\title{
Fluorescence Lifetime Imaging with a Blue Picosecond Diode Laser
}

\author{
D.S. Elson, S.E.D. Webb, J. Siegel, S. Lévêque-Fort and P.M.W. French \\ Photonics Group, Physics Dept., Imperial College, London SW7 2BW, U.K. \\ Tel: 44-20-7594 7706, Fax: 44-20-7594 7714, paul.french@ic.ac.uk \\ C. Anker and M. J. Lever \\ Department of Bioengineering, Imperial College, London SW7 2BY, U.K. \\ Tel: 44-20-7594 5172, m.j.lever@ic.ac.uk
}

K. Lauritsen, M. Wahl and R. Erdmann

PicoQuant GmbH, Rudower Chaussee 29, 12489 Berlin, Germany

Tel: 49-30-6392-6560,wahl@pq.fta-berlin.de

\begin{abstract}
Use of a blue picosecond laser diode allows a portable fluorescence lifetime imaging system. We show the application of the system to multi-well plate imaging of biological fluorophores and microscopic imaging of unstained tissue sections.

(C)2002 Optical Society of America

OCIS codes: $170.2520,170.6920$
\end{abstract}

Fluorescence permits both the monitoring of specific endogenous and/or exogenous fluorophores and the study of anatomical features. Fluorescence lifetime is the average decay time of the fluorescence after excitation with an ultrashort laser pulse. The determination of fluorescence lifetime is independent of intensity and fluorophore concentration, providing a robust means of extracting both functional and structural information. This can result from the presence of specific fluorophores and from the dependence of fluorescence lifetime on local environmental factors (such as oxygen, $\left[\mathrm{Ca}^{2+}\right]$, pH etc.) e.g. [1]. Fluorescence Lifetime Imaging (FLIM) can thus provide noninvasive functional/diagnostic imaging and a method for wide-field assays of distributed fluorophores. FLIM may be implemented in both a microscope, suited to studying tissue sections, and a macroscopic-imaging system for multi-well plate assays. To date, a major limitation in the implementation of time-domain fluorescence lifetime technology has been the complexity, cost and size of the laser excitation source. We have developed time-domain FLIM instruments based on an argon ion-pumped Ti:Sapphire laser [2], on an all-solid-state direct diode-pumped Cr:LiSAF laser system [3] and, most recently, a blue picosecond pulsed diode laser. To our knowledge, the latter represents the first such instrument, demonstrating a dramatic reduction in cost and complexity and reducing the footprint of the instrument to $<0.2 \mathrm{~m}^{2}$, providing portable time-domain FLIM technology. Furthermore, we show that the adjustable repetition rate of the diode laser provides an important advantage over conventional mode-locked solid-state lasers.

(a)

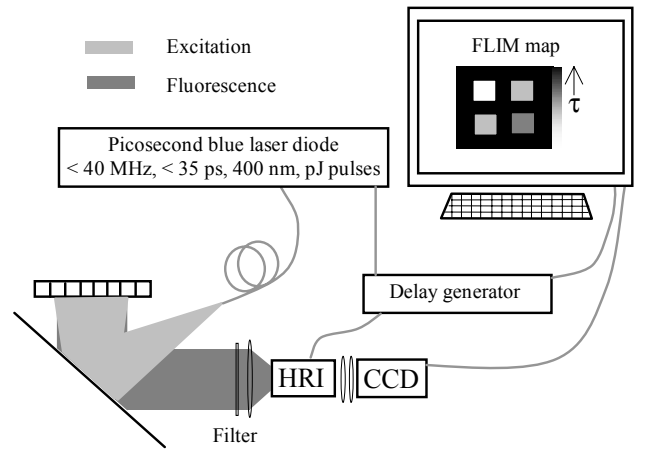

(b)

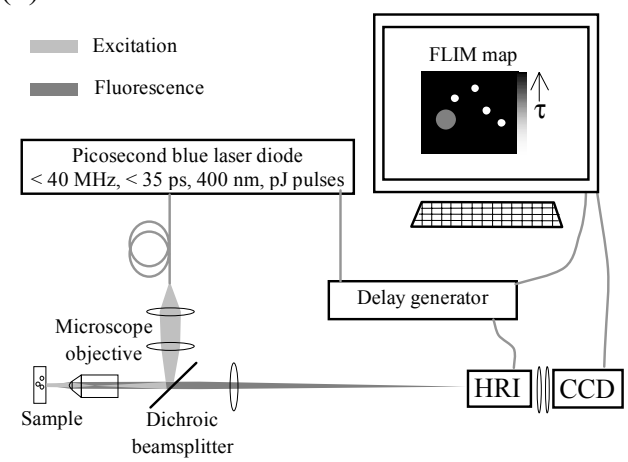

Figure 1. Experimental set-up for (a) macro-imaging and (b) microscopic imaging. 
The picosecond blue diode laser has been used to macro-image multi-well plates containing selections of chemically and biologically significant fluorophores. The output of the diode is coupled into a $62.5 \mu \mathrm{m}$ core diameter, 0.275 NA optical fibre, the divergent output of which is used to illuminate the sample (see figure 1(a)). The emitted fluorescence was imaged onto an ultrafast-gated optical intensifier (HRI) whose phosphor screen was read out by a CCD camera. By recording several frames at different temporal positions of the gate (width $\geq 200 \mathrm{ps)}$ of the HRI with respect to the excitation pulse, the fluorescence decay can be sampled. This wide-field acquisition yields a series of time-gated images. By performing a least-squares exponential fit for each pixel in the field of view and by plotting the resulting decay constant (fluorescence lifetime), a FLIM map is obtained. The maximum measurable lifetime is dependent only on the repetition rate of the laser system $(5 \mathrm{KHz}$ for the diode-pumped Cr:LiSAF oscillator/amplifier, $80 \mathrm{MHz}$ for the argon ion-pumped Ti:Sapphire laser and $2-40 \mathrm{MHz}$ for the blue picosecond diode laser). Figure 2(a) shows a FLIM map of 5 wells from a 384 well plate containing five biologically interesting fluorophores - protoporphyrin IX, $\beta$-reduced nicotinamide adenine dinucleotide ( $\beta$-NADH), collagen (from bovine Achilles tendon), elastin (from bovine neck) and $\beta$-flavin adenine dinucleotide. Collagen and elastin are the two most important structural proteins in tissue. Porphyrins are known to accumulate preferentially in various cancerous tumours, while NADH and flavins are two fluorophores associated with cellular metabolism. While these fluorophores exhibit different emission spectra [4], the considerable spectral overlap between the different chemical species makes the fluorescence lifetime a valuable extra parameter when distinguishing between them. For example, the emission spectra for collagen and elastin with $400 \mathrm{~nm}$ excitation are almost indistinguishable.

The laser has also been implemented as the illumination source in an inverted epi-illumination microscope that has been used to image test samples of biological tissue sections, an example which is shown in figure 2(b). The image shows an unstained $15 \mu \mathrm{m}$ thick section of a rabbit abdominal artery wall at x100 magnification. The highly convolved elastin-rich fibres are clearly seen with a longer lifetime than the surrounding tissue $(\sim 2.4 \mathrm{~ns})$.
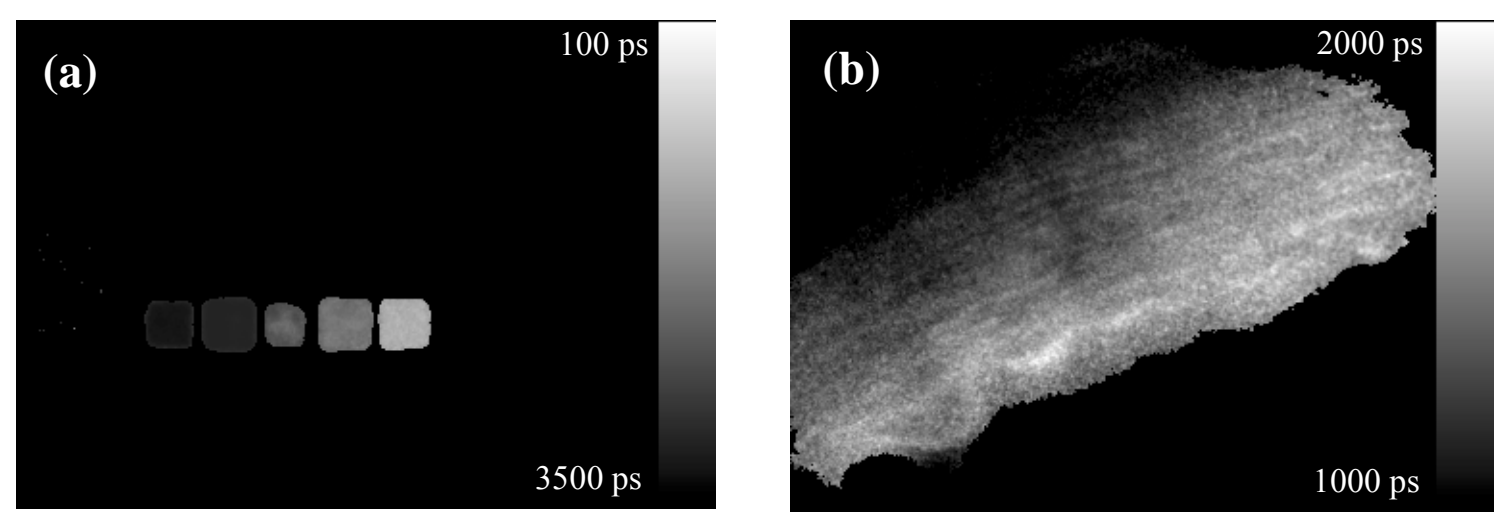

Figure 2. (a) Multi-well plate FLIM map of (left to right) porphyrins, NADH, collagen, elastin and flavins. (b) Microscope FLIM map of an unstained section of a rabbit abdominal artery wall.

We have studied the effect that the repetition rate has on the lifetime maps calculated and have observed that too short a period between the excitation pulses, compared to the lifetimes being measured, results in degraded FLIM maps exhibiting more noise and reporting lifetime values that are too low. This arises from the fact that the background intensity is treated as a free parameter in the fitting algorithm. For too short a sampling range the fitted background will significantly differ from the true background and will fluctuate statistically, introducing additional noise in the calculated lifetime values. There are many samples for which the $80 \mathrm{MHz}$ output from "standard" mode-locked solid-state lasers cannot sample the fluorescence intensities at longer delays. Lower repetition rate sources are clearly more widely applicable and the flexibility available from ultrafast-pulsed diode lasers with adjustable repetition rates is particularly useful.

There are many situations where it is not possible to contrast different fluorophores sufficiently by their fluorescence lifetime alone, in which case spectral information may provide additional discrimination. We have recently combined spectral resolution and lifetime imaging, simultaneously imaging fluorescence in two different 
spectral bands on a single wide-field time-gated detector [5]. This can be realized using a commercially available wide-field configuration of dichroic mirrors and filters. As a result, two FLIM maps that correspond to identical spatial regions of the sample but represent the lifetime maps of two different emission bands are obtained with a single acquisition run. This can provide a characteristic spectral/lifetime signature for a specimen, e.g. for biopsy of biological tissue, and can facilitate a wide range of fluorescence techniques, e.g. for cell biology and assays. We have applied this technique to study this spectral/lifetime signature in a sample of fresh rat heart [5] using the diodepumped Cr:LiSAF laser system and have observed significantly different lifetime values for the blue, green and red spectral regions of the fluorescence. We note that multi-spectral FLIM can be readily implemented with the pulsed diode laser to provide a powerful portable robust instrument. Furthermore, it can be extended to realise optical sectioning using structured illumination [5] and thereby provide a 5-D wide-field fluorescence imaging instrument.

\section{References:}

[1] J.R. Lakowicz, "Principles of Fluorescence Spectroscopy", $2^{\text {nd }}$ ed., Plenum Press (1999)

[2] S. E. D. Webb, Y. Gu, S. Lévêque-Fort, J. Siegel, M. J. Cole, K. Dowling, R. Jones, M. J. Lever, M. A. A. Neil, R. Juškaitis, L. O. Sucharov, T. Wilson, and P. M. W. French, Submitted for publication, Rev. Sci. Inst.

[3] R. Jones, K. Dowling, M. J. Cole, D. Parsons-Karavassilis, M. J. Lever, P. M. W. French, J. D. Hares and A. K. L. Dymoke-Bradshaw, Electron. Lett. 35, 256-258 (1999)

[4] G. A. Wagnières, W. M. Star and B. C. Wilson, "In vivo fluorescence spectroscopy and imaging for oncological applications", Photochem. Photobiol. 68, (1998) 603

[5] J. Siegel, D. S. Elson, S. E. D. Webb, D. Parsons-Karavassilis, S. Leveque-Fort, M. J. Cole, M. J. Lever, P. M. W. French, M. A. A. Neil, R. Juskaitis, L. O. Sucharov and T. Wilson, Opt. Lett. 26, 1338-1340 (2001) 